\title{
Green-emitting MADF complex for OLED applications
}

Kody Klimes, Zhi-Qiang Zhu, Sean Holloway, Jian Li

Kody Klimes, Zhi-Qiang Zhu, Sean Holloway, Jian Li, "Green-emitting MADF complex for OLED applications," Proc. SPIE 9941, Organic Light Emitting Materials and Devices XX, 99410L (23 September 2016); doi:

$10.1117 / 12.2244676$

Event: SPIE Organic Photonics + Electronics, 2016, San Diego, California, United States 


\title{
Green-Emitting MADF Complex for OLED Applications
}

\author{
Kody Klimes, Zhi-Qiang Zhu, Sean Holloway, Jian Li \\ Material Science and Engineering, Arizona State University, Tempe, Arizona 85287,United States
}

\begin{abstract}
In this article, we demonstrated an exceptional palladium complex that exhibits both phosphorescence and delayed fluorescence for use as an efficient emitter in OLEDs. Devices employing PdN3N achieved external quantum efficiencies in excess of $22 \%$ and remarkable device operational lifetime to $90 \%$ initial luminance estimated at over $30,000 \mathrm{~h}$ at a practical luminance of $100 \mathrm{~cd} / \mathrm{m}^{2}$. Further tuning of the phosphorescent and delayed fluorescent emission should have a great impact in the development of efficient and stable emitters for deep blue or white OLEDs.
\end{abstract}

Keywords: MADF, OLED, Pd Complexes, Blue

\section{INTRODUCTION}

In recent years organic light emitting diodes (OLEDs) have widely impacted the commercial marketplace, with applications ranging from next generation displays to solid-state lighting. ${ }^{1}$ This has been made possible as a result of the development materials and efficient OLED devices with emission profiles spanning the visible spectrum. ${ }^{2}$ Despite the advancements made in the field of organic electronics, there remain a number of key hurdles that separate OLEDs from becoming a widely adopted technology. Among these hurdles is the development of efficient and stable blue emissive compounds. ${ }^{3}$ While a small number of groups have demonstrated deep blue devices whose efficiency is on par with its "green" and "red" analogs, none of these reports provide an immediate solution because of their hindered device operational stability. ${ }^{4}$ Additionally, the formation of triplet excitons is speculated to directly facilitate the dissociation of $\sigma$-bonds. This concept has been demonstrated on the Si-Si bond in polysilane materials as well as other material systems reinforcing the challenges of developing stable deep blue triplet emitters. ${ }^{5}$ Assessing these shortcomings from the energy standpoint, the ideal approach entails the development of efficient blue emitters capable of harvesting all of the "blue" singlet and triplet excitons while having the triplet energy in the "green" or "red" region. This approach is beyond the reach of current blue fluorescent emitters, which cannot harvest triplet excitons, and green phosphorescent emitters, which cannot emit in the blue region. Thus a newly developed molecular design will be required to clear this hurdle.

Over the past few decades the detailed mechanisms for harvesting electro-generated excitons inside organic electroluminescent devices has been extensively studied and well documented. ${ }^{6}$ For typical florescent emitters their main radiative decay process is through the fluorescent pathway while the phosphorescent pathway is suppressed due to its symmetry forbidden character. Alternatively cyclometalated Ir and Pt complexes exhibit a rapid phosphorescence process that results from fast intersystem crossing due to strong spin-orbit coupling. This allows them to harvest both electro-generated singlet and triplet excitons, resulting in a theoretical $100 \%$ electron to photon conversion efficiency. ${ }^{7}$ Recent studies on carbazolyl-dicyanobenzene derivatives and copper(I) based metal complexes has characterized them as thermally activated delayed fluorescent emitters. ${ }^{8}$ These emitters are capable to harvest both electro-generated singlet and triplet excitons while exhibiting high emission quantum yield at room temperature. ${ }^{9}$ Figure 1 illustrates the energy transfer processes for TADF emitters. When the lowest triplet excited state (T1) and the lowest singlet excited state $\left(\mathrm{S}_{1}\right)$ share similar energy levels, the triplet excitons are capable of radiative decay though both intersystem crossing $\left(\mathrm{T}_{1} \rightarrow \mathrm{S}_{1}\right)$ and delayed fluorescence $\left(\mathrm{S}_{1} \rightarrow \mathrm{S}_{0}\right)$. As a result this materials can exhibit higher energy emission while having a triplet energy that is comparably lower, this leads to emitters compatible with known stable host and transport materials unlike many deep blue Ir or Pt emitters. ${ }^{10}$ Nonetheless, this is an endothermic process and a portion of the triplet excitons will decay non-radiativly due to the absence of an efficient phosphorescent emission pathway. ${ }^{11}$ This results in TADF emitters only being able to achieve high efficiencies for very small $\mathrm{S}_{1}-\mathrm{T}_{1}$ energy gaps. ${ }^{12}$ 


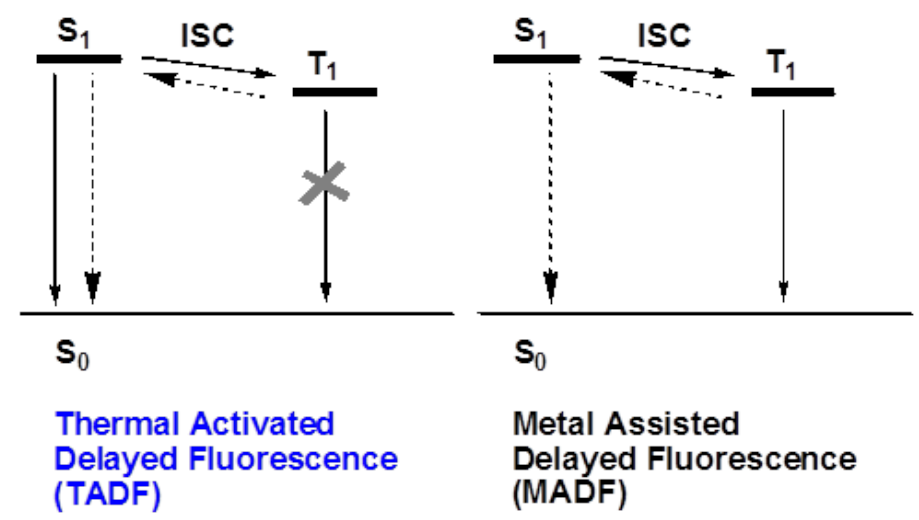

Figure 1. Illustration of the various emission mechanisms for organic emitters.

In this article, we will demonstrate another mechanism of utilizing electro-generated excitons, this process is denoted as metal-assisted delayed fluorescence (MADF), where a heavy metal ion will be incorporated into the complex system to ensure both efficient phosphorescence and delayed fluorescence processes. As proposed in Fig. 1, when the energy levels of the $T_{1}$ state and the $S_{1}$ state are reasonably close, the two radiative decay process, i.e. phosphorescence $\left(T_{1} \rightarrow S_{0}\right)$ and thermally activated delayed fluorescence $\left(\mathrm{S}_{1} \rightarrow \mathrm{S}_{0}\right)$ can potentially occur simultaneously, enabling dual emission pathways. Due to its efficient triplet emission process, the MADF emitters can harvest all of singlet and triplet excitons regardless of a larger energy difference between the $T_{1}$ and $S_{1}$ states.

We have synthesized and characterized one green emitting palladium complexe, i.e. PdN3N, which exhibit both efficient phosphorescence and delayed fluorescence processes. Devices of PdN3N achieved over $22 \%$ peak external quantum efficiency (EQE) and also demonstrated remarkable device operational stability to $90 \%$ initial luminance $\left(\mathrm{LT}_{90}\right)$ estimated at over $30,000 \mathrm{hrs}$ at $100 \mathrm{~cd} / \mathrm{m}^{2}$.

\section{PHOTOPHYSICAL STUDIES}

The absorption spectra for PdN3N as well as the N3N ligand are shown in Figure $2 .{ }^{13}$ Both the complex and ligand exhibit very strong absorption bands below $400 \mathrm{~nm}\left(\varepsilon>10^{4} \mathrm{~cm}^{-1}\right)$ assigned to ${ }^{1} \pi-\pi^{*}$ transitions, localized on the cyclometalating ligands. The small shift to lower energy of these transitions in the complex is attributed to the preferable planar molecular geometry of the ligand when covalently bonded to the Pd ion as well as the anionic nature of the ligand in the complex. The intense bands in the 400-500 nm region $\left(\varepsilon \approx 10^{3}-10^{4} \mathrm{~cm}^{-1} \mathrm{M}^{-1}\right)$ are red shifted relative to all the absorption bands attributed to the ligand and are assigned to singlet metal to ligand charge transfer $\left({ }^{1} \mathrm{MLCT}\right)$ transitions.

The $77 \mathrm{~K}$ emission spectrum shows a narrow primary emission peak at $522 \mathrm{~nm}$ with the small vibronic peaks characteristic of many phosphorescent emitters. The emission at $522 \mathrm{~nm}$ is attributed $\mathrm{T}_{1} \rightarrow \mathrm{S}_{0}$ transition on the basis of the large Stokes shift from the absorption cut-off. This has a longer wavelength than many existing phenyl-pyridine based complexes due to its extended conjugation through the carbazole units. ${ }^{14}$ At room temperature $(300 \mathrm{~K})$ the dominant emission peak is slightly red shifted to $534 \mathrm{~nm}$ and significantly broadened as is typically seen at elevated temperatures for most phosphorescent emitters. Unlike previously reported heavy metal phosphorescent complexes, a shorter wavelength peak appears $(\sim 505 \mathrm{~nm})$ at the elevated temperatures indicating the presence of a thermally activated emission process. By comparing the absorption and emission spectra of this complex, the higher energy emission process can be attributed to a $\mathrm{S}_{1} \rightarrow \mathrm{S}_{0}$ transition (fluorescence) on the basis of the relatively small stokes shift. The delayed fluorescent nature of this thermally activated emission peak is further supported by the comparable phosphorescent lifetimes of $142 \mu \mathrm{s}$ and $145 \mu \mathrm{s}$ when measured at detector wavelengths of $530 \mathrm{~nm}$ and $485 \mathrm{~nm}$ respectively. Thus, this demonstrates the existence of both phosphorescence and thermally activated delayed fluorescence within the single emitter. 
In order to model the relative phosphorescent and fluorescent contributions to the emission, Gaussian oscillators were fit to the emission spectrum on a linear energy axis. The phosphorescence was fit with three transitions: $\mathrm{v}_{0-0}$ being the highest energy and the highest intensity, $\mathrm{v}_{0-1}$ being the first vibronic progressions and $\mathrm{v}_{0-2}$ being the second vibronic progressions as is characteristic for many aromatic cyclometalated Pt complexes. ${ }^{15}$ The shoulder attributed to fluorescent emission was fit with a single oscillator. The ratio of the fluorescent emission peak to the $\mathrm{v}_{0-0}$ phosphorescent transition is 0.56 and fluorescence is estimated to be responsible for roughly $25 \%$ of the total emission but more measurements are necessary to definitively determine this contribution. In the inset to Figure $2 \mathrm{~b}$, a CIE plot showing the approximate emission color of either pure phosphorescence, fluorescence, or the combination of the two. The dramatic shift in the $\mathrm{CIE}_{\mathrm{X}}$ values for the pure fluorescent emission highlights the value of the relatively large $\mathrm{T}_{1}-\mathrm{S}_{1}$ gap to achieve blue emission from an emitter with triplet energy in the "green" region. Further materials development of MADF emitters to increase the emission energy or increase the fluorescence to phosphorescence ratio is of the utmost importance for this goal.
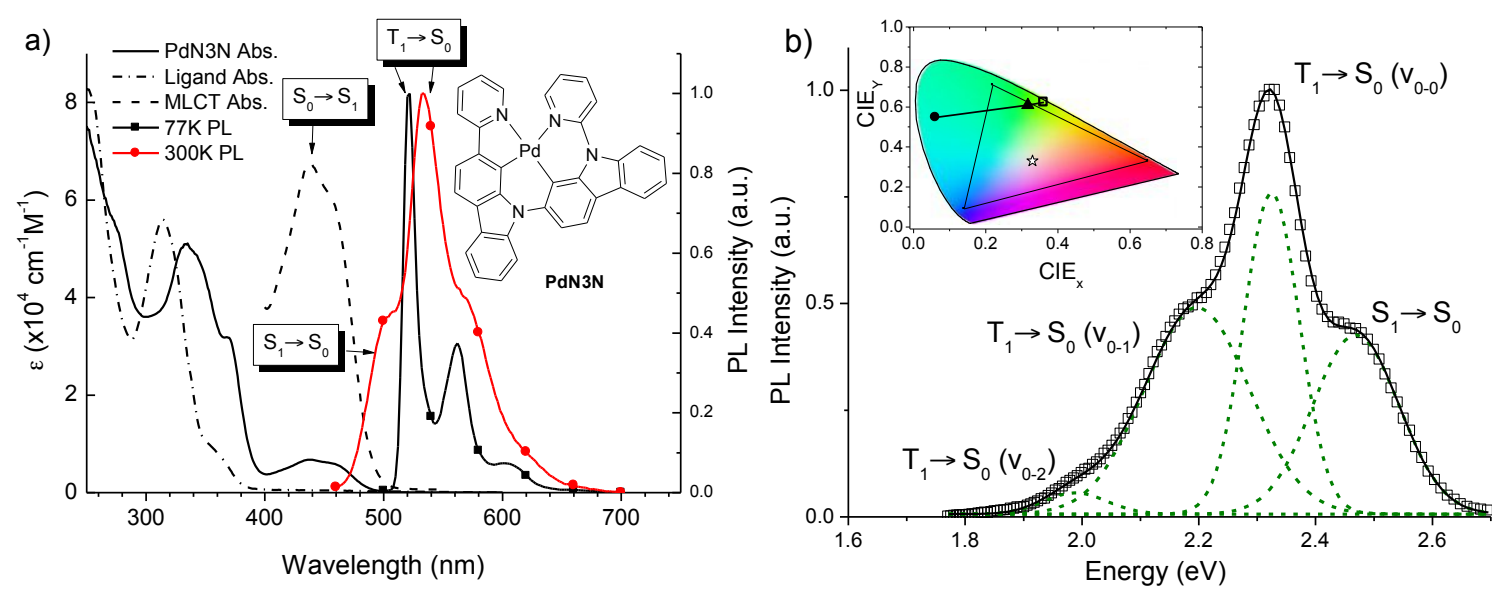

Figure 2. a) The extinction coefficient of Ligand N3N (dash-dot) and PdN3N (solid) and in a solution of dichloromethane, the PL spectrum at $77 \mathrm{~K}$ in a solution of $2-\mathrm{Me}-\mathrm{THF}$ (squares), and $300 \mathrm{~K}$ in a solution of $\mathrm{CH}_{2} \mathrm{Cl}_{2}$ (circles). The MLCT character of the absorption spectrum is enlarged for clarity (dashed line). b) PL spectrum vs. energy (squares) with Gaussian oscillator fits (solid line). CIE coordinates of spectra for fluorescent only (solid circle), phosphorescent only (open square, and combined (triangle) are given in the inset.

To explore the origins and characteristics of the MADF, PL spectra as a function of temperature was measured for PdN3N, shown in Figure 3. The designed compound can be described as containing two parts: a $\mathrm{C}^{\wedge} \mathrm{N}$ cyclometalating ligand containing the pyridyl-carbazole portion of the molecules and the donor-acceptor (D-A) - portion of either cabazole-phenoxyl-pyridine or carbazole-carbazolyl-pyridine. The triplet state consists mostly of the lower energy $\mathrm{C}^{\wedge} \mathrm{N}$ portion of the molecules which is localized on the pyridyl-carbazole and as such the phosphorescent peak at $77 \mathrm{~K}$ is approximately PdN3N $(522 \mathrm{~nm})$. As temperature increases the thermally activated delayed fluorescence peak increases in intensity relative to the phosphorescent peak.

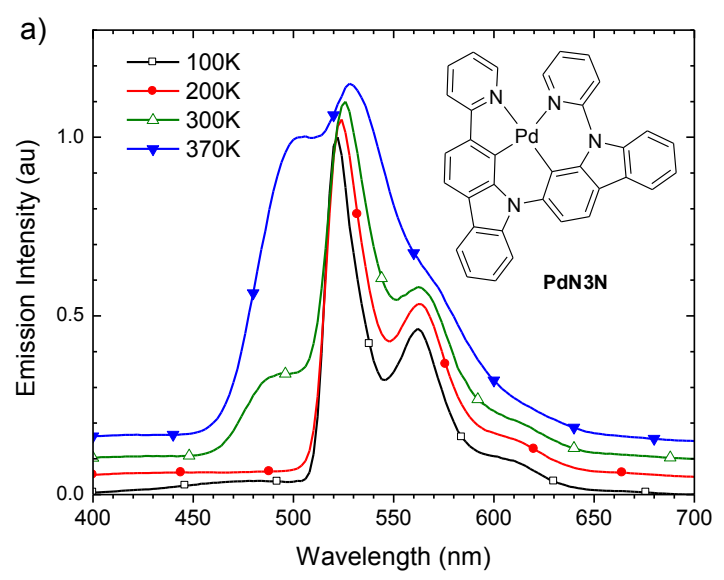

Figure 3. The PL spectrum as a function of temperature for a) PdN3N and b) PdN3O doped 6\% (wt/wt) in PMMA film. 


\section{DEVICE PERFORMANCE}

To explore the device performance of this novel MADF emitter, OLED devices were fabricated on glass substrates pre-coated with a patterned layer of indium tin oxide (ITO) to serve as the transparent anode for the structure ITO/HATCN(10 nm)/NPD(30 nm)/TAPC(10 nm)/6\% dopant: 26mCPy $(25 \mathrm{~nm}) / \mathrm{DPPS}(10 \mathrm{~nm}) / \mathrm{BmPyPB}(40 \mathrm{~nm}) / \mathrm{LiF} / \mathrm{Al}$ where HATCN is 1,4,5,8,9,11-hexaazatriphenylene-hexacarbonitrile, NPD is N,N'-diphenyl-N,N'-bis(1-naphthyl)-1,1'biphenyl-4,4"-diamine, TAPC is di-[4-(N,N-di-tolyl-amino)-phenyl]cyclohexane, 26mCPy is 2,6-bis(N-carbazolyl) pyridine, DPPS is diphenyl-bis[4-(pyridin-3-yl)phenyl]silane, and BmPyPB is 1,3-bis[3, 5-di(pyridin-3yl)phenyl]benzene, this architecture will be referred to as structure one. ${ }^{16}$ The EQE is shown in Figure 4 with the electroluminescent spectrum inset. The EQE of PdN3N peaked at $20.9 \%$, which is among the highest achieved for any $\mathrm{Pd}$ complex and is comparable to many of the Ir and Pt analogs in a similar device setting. ${ }^{17}$ One apparent drawback to devices employing these complexes is the large efficiency roll off at higher luminance dropping to $14.3 \%$ at $100 \mathrm{~cd} / \mathrm{m}^{2}$. This is attributed to a combination of poor charge balance due to the poor electron transporting properties of DPPS and the long phosphorescent lifetime of $142 \mu \mathrm{s}$. This issue can be addressed through improved charge balance or by improving the spontaneous radiative decay rate as has been demonstrated previously employing more advanced structures such as plasmonic nanostructures or microcavities. ${ }^{18}$

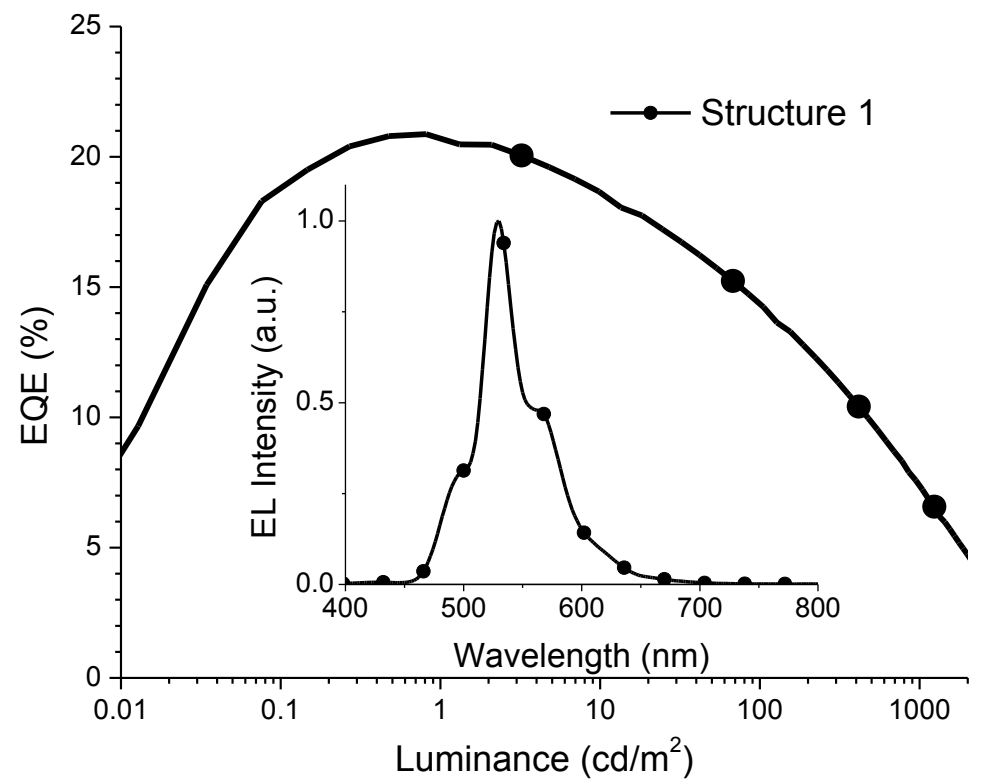

Figure 4. EQE versus Luminance with the EL spectra at $1 \mathrm{~mA} / \mathrm{cm}^{2}$ (inset) for devices with the structure: ITO/HATCN/NPD/TAPC/6\% PdN3N: 26mCPy/DPPS/BmPyPB/LiF/Al.

The electroluminescent characteristics show both fluorescent and phosphorescent emission in the devices. PdN3N devices showed a delayed fluorescence peak of approximately $30 \%$ of the phosphorescence peak height but the color was still dominantly from the green phosphorescent emission spectrum with CIE $(0.30,0.61)$. The high emission efficiency despite the moderate $\mathrm{T}_{1}-\mathrm{S}_{1}$ splitting, highlights the benefit of MADF emitters over their organic TADF counterparts. Excitons that would otherwise be unable to overcome the $S_{1}-T_{1}$ energy barrier can still emit through the efficient phosphorescent radiative pathway. Furthermore, alterations to the molecular structure can be used to adjust the relative height of the delayed fluorescence peak and shift the phosphorescent emission profile leading to even more ideal spectra.

In order to further explore the potential application of this Pd based MADF complex as stable emitter, two PdN3N devices were fabricated using known stable materials, structure two is ITO/HATCN $(10 \mathrm{~nm}) / \mathrm{NPD}(40 \mathrm{~nm}) / 10 \%$ PdN3N: CBP $(25 \mathrm{~nm}) /$ BAlq $(10 \mathrm{~nm}) / \mathrm{Alq}(30 \mathrm{~nm}) / \mathrm{LiF} / \mathrm{Al}$ where CBP is 4,4'-bis(N-carbazolyl) biphenyl, BAlq is bis(2-methyl-8quinolinolato) (biphenyl-4-olato)aluminum and Alq is tris-(8-hydroxyquinoline) aluminum. ${ }^{19}$ Structure three is 
ITO/HATCN (10 nm)/NPD (40nm)/Tris-PCz (10nm)/6\% PdN3N: CBP (25nm)/BAlq (10nm)/BPyTP (40nm)/LiF/Al

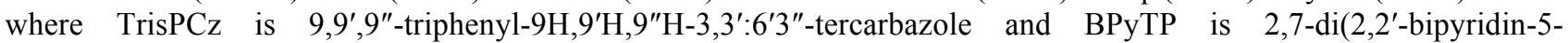
yl)triphenylene. The device performances of these structures are crucial to determining the viability of MADF complexes as a potential solution to high-energy stable emitters. Structure 2 exhibits a drastic reduction in device efficiency as a result of poor charge confinement and hindered electron mobility. Despite this drawback we demonstrated exceptional accelerated lifetime. The device was driven at a constant current of $20 \mathrm{~mA} / \mathrm{cm}^{2}$ corresponding to an initial luminance of approximately $1700 \mathrm{~cd} / \mathrm{m}^{2}$. The lifetime to $90 \%$ of the initial luminance at these elevated conditions was $170 \mathrm{~h}$ which translates to over $20,000 \mathrm{~h}$ at $100 \mathrm{~cd} / \mathrm{m}^{2}$ using the conversion equation $\mathrm{LT}\left(\mathrm{L}_{1}\right)=\mathrm{LT}\left(\mathrm{L}_{0}\right) *\left(\mathrm{~L}_{0} / \mathrm{L}_{1}\right){ }^{1.7} \cdot{ }^{20}$ Structure 3 address the shortcomings of the previous devices by implementing an electron blocking layer to confine excition recombination and by using an improved electron transport material. The EQE peaked at $22.1 \%$ exceeding the previous demonstration of efficiency. Additionally the high roll-off observed in structure one has been improved, the device maintained $17 \%$ EQE at $100 \mathrm{~cd} / \mathrm{m}^{2}$. Finally under accelerated decay at $20 \mathrm{~mA} / \mathrm{cm}^{2}$ and the initial luminance of approximately $3300 \mathrm{~cd} / \mathrm{m}^{2}$ exhibited a lifetime to $90 \%$ of the initial luminance of $78 \mathrm{~h}$ which translates to over $30,000 \mathrm{~h}$ at $100 \mathrm{~cd} / \mathrm{m}^{2}$.
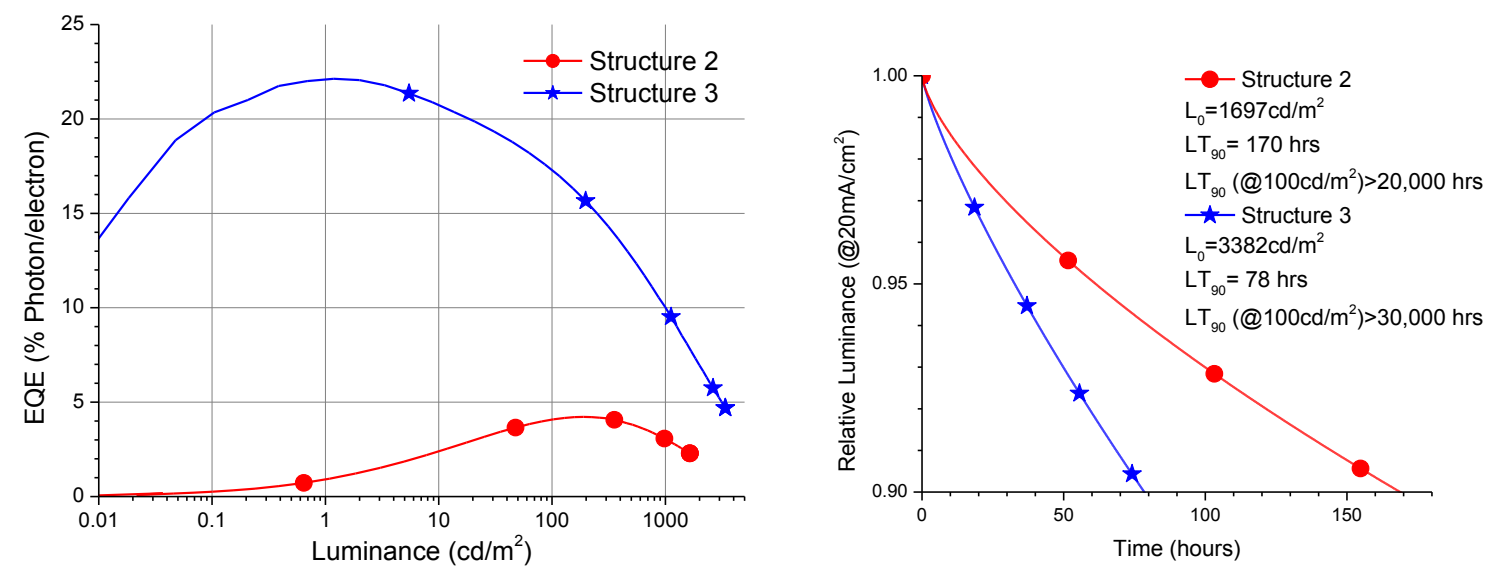

Figure 5. EQE and device operational lifetime at a constant driving current of $20 \mathrm{~mA} / \mathrm{cm}^{2}$ for Structure 2: ITO/HATCN/NPD/6\% PdN3N:CBP/BAlq/Alq/LiF/Al and structure 3: ITO/HATCN/NPD/TrisPCz/6\%PdN3N:CBP/BAlq/BPyTP/ LiF/A

\section{CONCLUSIONS}

In this article, we demonstrated an exceptional palladium complex that exhibits both phosphorescence and delayed fluorescence for use as an efficient emitter in OLEDs. Devices employing PdN3N achieved external quantum efficiencies in excess of $22 \%$ and remarkable device operational lifetime to $90 \%$ initial luminance estimated at over $30,000 \mathrm{~h}$ at a practical luminance of $100 \mathrm{~cd} / \mathrm{m}^{2}$. Further tuning of the phosphorescent and delayed fluorescent emission should have a great impact in the development of efficient and stable emitters for deep blue or white OLEDs.

\section{REFERENCES}

1 a) S. R. Forrest, Nature 2004, 428, 911-918; b) L. Xiao, Z. Chen, B. Qu, J. Luo, S. Kong, Q. Gong, J. Kido, Adv. Mater. 2011, 23, 926-952.

2 a) M. K. Nazeeruddin, R. Humphry-Baker, D. Berner, S. Rivier, L. Zuppiroli, M. Graetzel, J. Am. Chem. Soc. 2003, 125, 8790-8797; b) N. R. Evans, L. S. Devi, C. S. K. Mak, S. E. Watkins, S. I. Pascu, A. Köhler, R. H. Friend, C. K. Williams, A. B. Holmes, J. Am. Chem. Soc. 2006, 128, 6647- 6656; c) V. K. Au, K. M. Wong, D. P. Tsang, M. -Y. Chan, N. Zhu, V. W. Yam, J. Am. Chem. Soc. 2010, 132, 14273-14278; d) C. -C. Hsu, C. -C. Lin, P. -T. Chou, C. -H. Lai, C. -W. Hsu, C. -H. Lin, Y. Chi, J. Am. Chem. Soc. 2012, 134, 7715-7724; e) X. Yang, D. C. Müller, D. Neher, K. Meerholz, Adv. Mater. 2006, 18, 948-954; f) S. -C. Lo, R. E. Harding, C. P. Shipley, S. G. Stevenson, P. L. Burn, I. D. 
W. Samuel, J. Am. Chem. Soc. 2009, 131, 16681-16688; g) T. Qin, J. Ding, L. Wang, M. Baumgarten, G. Zhou, K. Müllen, J. Am. Chem. Soc. 2009, 131, 14329-14336; h) Y. You, S. Y. Park, J. Am. Chem. Soc. 2005, 127, 12438-12439; i) C. -M. Che, C. -C. Kwok, S. -W. Lai, A. F. Rausch, W. J. Finkenzeller, N. Zhu, H. Yersin, Chem. Eur. J. 2010, 16, 233-247; j) G. Li, T. Fleetham, J. Li, Adv. Mater. 2014, DOI: 10.1002/adma.201305507; k) G. Cheng, K. T. Chang, W. P. To, C. -M. Che, Adv. Mater. 2014, 26, 2540-2546.

${ }^{3}$ a) K. S. Yook, J. Y. Lee, Adv. Mater. 2012, 24, 3169-3190; b) M. C. Gather, A. Kohnen, K. Meerholz, Adv. Mater. 2011, 23, 233-248.

4 a) T. Fleetham, Z. Wang, J. Li, Org. Electron. 2012, 13, 1430-1435; b) X. -C. Hang, T. Fleetham, E. Turner, J. Brooks, J. Li, Angew. Chem Int. Ed. 2013, 52, 6753-6756; c) S. Lee, S. -O. Kim, H. Shin, H. -J. Yun, K. Yang, S. -K. Kwon, J. -J. Kim, Y. -H. Kim, J. Am. Chem. Soc. 2013, 135, 14321-14328; d) Z. M. Hudson, C. Sun, M. G. Helander, Y. -L. Chang, Z. -H. Lu, S. Wang, J. Am. Chem. Soc. 2012, 134, 13930-13933; e) K. Li, G. Cheng, C. Ma, X. Guan, W. -M. Kwok, Y. Chen, W. Lu, C. -M. Che, Chem. Sci. 2013, 4, 2630-2644.

${ }^{5}$ J. Michl, Acc. Chem. Res. 1990, 23, 127-128.

${ }^{6}$ a) N. J. Turo, Modern Molecular Photochemistry. University Science Books, Mill Valley, CA, 1991; b) M. A. Baldo, S. Lamansky, P. E. Burrows, M. E. Thompson, S. R. Forrest, Appl. Phys. Lett. 1999, 75, 4-6; c) M. A. Baldo, M. E. Thompson, S. R. Forrest, Nature 2000, 403, 750-753.

7 a) M. A. Baldo, D. F. O’Brien, Y. You, A. Shoustikov, S. Sibley, M. E. Thompson, S. R. Forrest, Nature 1998, 395, 151-154; b) C. Adachi, M. A. Baldo, S. R. Forrest, M. E. Thompson, Appl. Phys. Lett. 2000, 77, 904-906; c) Y.-S. Park, S. Lee, K.-H. Kim, S.-Y. Kim, J.-H. Lee, J.-J. Kim, Adv. Funct. Mater. 2013, 23, 4914-4920.

8 a) H. Uoyama, K. Goushi, K. Shizu, H. Nomura, C. Adachi, Nature 2012, 492, 234-238; b) F. B. Dias, K. N. Bourdakos, V. Jankus, K. C. Moss, K. T. Kamtekar, V. Bhalla, J. Santos, M. R. Bryce, A. P. Monkman, Adv. Mater. 2013, 25, 3707-3714;

9 a) Y. Im, J. Y. Lee, Chem. Mater. 2014, 26, 1413-1419; b) M. Osawa, Chem. Commun. 2014, 50, 1801-1803; c) D. M. Zink, M. Bächle, T. Baumann, M. Nieger, M. Kühn, C. Wang, W. Klopper, U. Monkowius, T. Hofbeck, H. Yersin, S. Bräse, Inorg. Chem. 2013, 52, 2292-2305.

${ }^{10}$ X. Ren, J. Li, R. J. Holmes, P. I. Djurovich, S. R. Forrest, M. E. Thompson, Chem. Mater. 2004, 16, 4743-4747.

${ }^{11}$ Q. Zhang, J. Li, K. Shizu, S. Huang, S. Hirata, H. Miyazaki, C. Adachi, J. Am. Chem. Soc. 2012, 134, 14706-14709

${ }^{12}$ Q. Zhang, B. Li, S. Huang, H. Nomura, H. Tanaka, C. Adachi, Nat. Photonics. 2014. 8. 326-332.

${ }^{13}$ Z. Q. Zhu, T. Fleetham, E. Turner, J. Li, Adv. Mater. 2015, 27, 2533-2537.

14 a) J. Kalinowski, V. Fattori, M. Cocchi, J. A. G. Williams, Coord. Chem. Rev. 2011, 255, 2401-2425; b) J. Brooks, Y. Babayan, S. Lamansky, P. I. Djurovich, I. Tsyba, R. Bau, M. E. Thompson, Inorg. Chem. 2002, 41, 3055-3066; c) D. A. K. Vezzu, J. C. Deaton, J. S. Jones, L. Bartolotti, C. F. Harris, A. P. Marchetti, M. Kondakova, R. D. Pike, S. Huo, Inorg. Chem. 2010, 49, 5107-5119.

${ }^{15}$ J. Li, P. I. Djurovich, B. D. Alleyne, M. Yousufuddin, N. N. Ho, J. C. Thomas, J. C. Peters, R. Bau, M. E. Thompson, Inorg. Chem. 2005, 44, 1713-1727.

${ }^{16}$ a) S. -J. Su, E. Gonmori, H. Sasabe, J. Kido, Adv. Mater. 2008, 20, 4189-4194; b) N. Chopra, J. S. Swensen, E. Polikarpov, L. Cosimbescu, F. So, A. B. Padmaperuma, Appl. Phys. Lett. 2010, 97, 033304. 
${ }^{17}$ P. K. Chow, C. Ma, W. -P. To, G. S. M. Tong, S. -L. Lai, S. C. F. Kui, W. -M. Kwok, C. -M. Che, Angew. Chem. Int. Ed. 2013, 52, $11775-11779$.

18 a) C. Murawski, K. Leo, M. C. Gather, Adv. Mater. 2013, 25, 6801-6827; b) M. G. Helander, Z. B. Wang, J. Qiu, M. T. Greiner, D. P. Puzzo, Z. W. Liu, Z. H. Lu, Science 2011, 332, 944-947; c) D. Tanaka, H. Sasabe, Y.-J. Li, S.-J. Su, T. Takeda, and J. Kido, Jpn. J. Appl. Phys. 2007, 46, L10.

${ }^{19}$ R. C. Kwong, M. R. Nugent, L. Michalski, T. Ngo, K. Rajan, Y. -J. Tung, M. S. Weaver, T. X. Zhou, M. Hack, M. E. Thompson, S. R. Forrest, J. J. Brown, Appl. Phys. Lett. 2002, 81, 162-164.

${ }^{20}$ C. Féry, B. Racine, D. Vaufrey, H. Doyeux, S. Cinà Appl. Phys. Lett. 2005, 87, 213502. 